\title{
Strain Release in Metastable CdSe/CdS Quantum Dots
}

\author{
Ke Gong, Gary Beane and David F. Kelley* \\ Chemistry and Chemical Biology, University of California, Merced, 5200 North Lake Road, \\ Merced, California 95343, USA
}

\begin{abstract}
It has recently been shown (J. Phys. Chem. Lett., 2015, 6, 1559) that high quantum yields (QYs) in zincblende $\mathrm{CdSe} / \mathrm{CdS}$ quantum dots can be achieved when the lattice strain energy density is in the stable $\left(0-0.59 \mathrm{eV} / \mathrm{nm}^{2}\right)$ or metastable $\left(0.59-0.85 \mathrm{eV} / \mathrm{nm}^{2}\right)$ regime. Annealing of metastable particles causes a dramatic reduction in the observed QY and a red shift of the absorbance and photoluminescence. In this work we demonstrate that the decline in QY upon annealing is due to the formation of hole traps. These traps, while dramatically affecting the observed QY, produce no significant changes in either morphology or crystallinity as determined by high resolution transmission electron microscopy (HRTEM).
\end{abstract}

*Corresponding author: dfkelley@ucmerced.edu

Keywords: core/shell interface, annealing, photoluminescence, quantum yield, shell morphology. 


\section{INTRODUCTION}

Core/shell semiconductor quantum dots (QDs) often yield much higher luminescence quantum yields (QYs) and better stabilities than the bare cores. The use of a higher bandgap semiconductor (e.g. ZnSe, ZnS or CdS) on a CdSe core produces a confinement potential which effectively isolates the hole wavefunction from the surface defect sites.[1-4] The trapping of the charge carriers on surface defect sites results in non-radiative recombination pathways and therefore photoluminescence QYs that are below unity.[5] In CdSe/CdS quantum dots - so called "quasi-type-II" - the conduction band offset is quite small (approximately 0 to $0.30 \mathrm{eV}$ ) while there is a considerable valence band offset $(0.6-0.7 \mathrm{eV})$. The result is that photogenerated holes are localized in the CdSe core and decoupled from surface trapping processes.

One important reason that CdS is a good shell material for passivation of CdSe QDs is that there is a small lattice mismatch, $\sim 4 \%$. This makes possible the deposition of several CdS monolayers without lattice strain induced irregular shell growth or the production of lattice strain induced defects. The presence of a shell having a smaller lattice parameter than the core also results in core compression, which raises the bandgap of the core material and thereby shifts the core-localized excitons to higher energies. Recently, we reported that uniform CdS shell growth occurs on a wurtzite CdSe core only until a critical thickness is reached.[6] Further shell growth causes sufficient lattice strain to make uniform shell growth thermodynamically unfavorable. If the strain energy exceeds some critical value, it is released by roughening of the surface and/or defect production. The same phenomenon has recently been described in the shell growth of other core/shell quantum dots, such as ZnTe/CdSe.[7, 8] The critical thickness, beyond which a uniform shell is no longer thermodynamically favored, is determined by the total energy of the particles, with contributions from the lattice strain and surface energies. The previous studies correlated the experimentally determined onset of irregular shell morphologies with the results of elastic continuum calculations.[6,9] The results indicate that for particles that are in thermodynamic equilibrium, irregular shells are formed when the strain energy density (total elastic strain energy of the particle divided by the core surface area) exceeds about $0.59 \mathrm{eV} / \mathrm{nm}^{2}$. This explains why high-quality "giant quantum dots" generally have lower PL QYs than their thin shell counterparts.[10-12] An important caveat to analysis is that the particle be in thermodynamic equilibrium. Very recently, we suggested that synthesis of CdSe/CdS particles that are not in thermodynamic equilibrium can allow the deposition of thicker shells without degrading the optical properties and lowering the PL QY.[13] This can be achieved by depositing the shell at a sufficiently low temperature that the equilibrium (irregular) morphology is not achieved. Nan et al.[14] first reported the synthesis and optical properties of zincblende CdSe/CdS core/shell particles in which the shells are deposited at very low temperatures $\left(\sim 140^{\circ} \mathrm{C}\right)$. These QDs have excellent optical properties and very high PL QYs for 
very thin shell growth, i.e. 85\% for $2 \sim 4 \mathrm{CdS}$ monolayers (ML) and $>50 \%$ for $8 \sim 10 \mathrm{CdS}$ ML. Thus, for these particles continued shell deposition results in a metastable core-shell morphology having a smooth shell surface with very few defects, but very high lattice strain energy. However, this approach does have its limitations. At some shell thickness, the lattice strain energy becomes sufficiently large that it will not maintain the smooth-shell state, causing a drop of the QY.[13] The intermediate region with strain density between 0.59 and $0.85 \mathrm{eV} / \mathrm{nm}^{2}$ corresponds to the "metastable regime". This metastable to unstable transition was examined in our recent work, which reports that high quantum yields are achieved as long as the lattice strain energy density is below about $0.85 \mathrm{eV} / \mathrm{nm}^{2}$.

Zincblende core/shell particles in the metastable regime will lose high QYs if annealed at a sufficiently high temperature that thermodynamic equilibrium is reached, $>210{ }^{\circ} \mathrm{C}$. Associated with relaxation to thermodynamic equilibrium is the partial loss of core compression, and this results in the absorption spectrum shifting to lower energies. However, it is still unclear what nonradiative mechanism causes the drop in QY. In this work, we have combined photoluminescence decay and transient absorption measurements to elucidate the mechanism of radiationless decay induced by annealing. We have also utilized high-resolution transmission electron microscopy (TEM) to determine the extent to which structural defects caused by strain release can be imaged directly.

\section{RESULTS AND DISCUSSION}

\section{A. Spectroscopic analysis}

Figure 1A shows the absorption and photoluminescence (PL) spectra of zincblende CdSe/CdS core/shell particles with 3.60 diameter CdSe cores and $1.20 \mathrm{~nm}$ thick CdS shells. The lowest energy exciton (1S $-1 S_{h}$ ) peak shifts from $567 \mathrm{~nm}$ to $604 \mathrm{~nm}$ upon shell deposition, indicating that the electron is delocalized into the shell.[15] Before annealing, these particles are calculated to have a strain energy density of $\sim 0.67 \mathrm{eV} / \mathrm{nm}^{2}$, which is in the metastable regime. Following annealing at $210{ }^{\circ} \mathrm{C}$ for 15 minutes the lowest energy exciton peak in the absorption spectrum and the peak of the PL spectrum red shift by $4.0 \mathrm{~nm}$. These redshifts indicate strain release at the core/shell interface. Accompanying the strain release, the PLQY decreases from $77 \%$ to $15 \%$, about of a factor of 5 . Figure $1 \mathrm{~B}$ shows that the PL decay of the particles before annealing is almost single-exponential with time constant of $22 \mathrm{~ns}$. No longer lived components of the PL decay are observed and this decay is assigned to the radiative lifetime. The PL decay after annealing has a much smaller initial amplitude and somewhat faster decay components in the first $10 \mathrm{~ns}$ (see inset of figure 1B). Figure 1C shows the bleach recovery kinetics obtained in a low-power transient absorption (TA) experiment. These kinetics indicate the rate of depopulation of $1 S$ conduction band electrons. The before-annealing TA kinetics are fit to a 490 
ps exponential decay component having an amplitude of $25 \%$ of the total and a $75 \%$ component that is essentially constant on this time scale. The 490 ps component is assigned to the Auger lifetime of the surface charged positive trion and is in good agreement with the decay time reported previously.[16] Following annealing the initial magnitude of the bleach decreases by about $25 \%$, far less than the factor of 5 decrease in the initial PL amplitude. The TA kinetics show the same 490 ps decay component, but its amplitude has decreased by about $30 \%$.
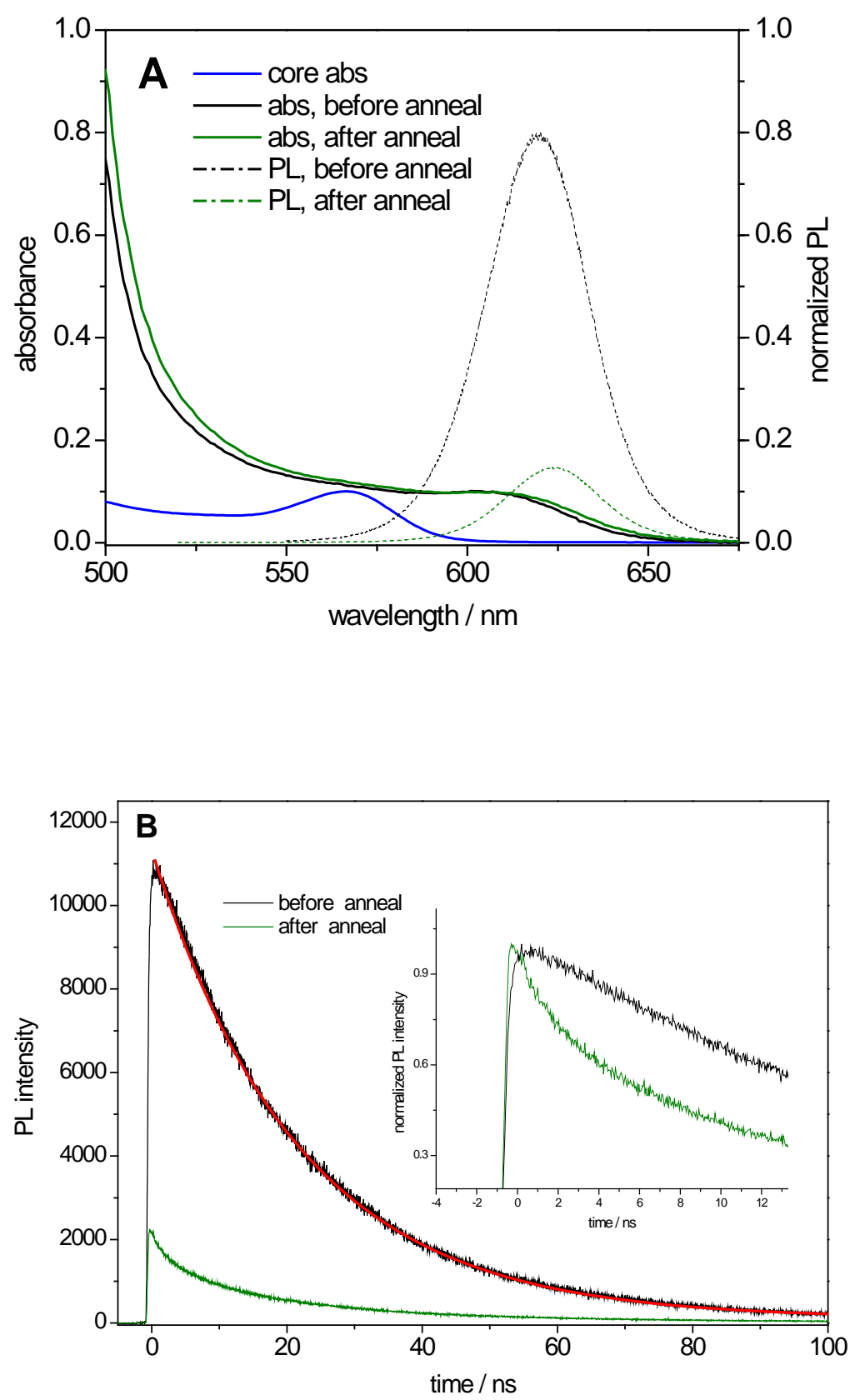


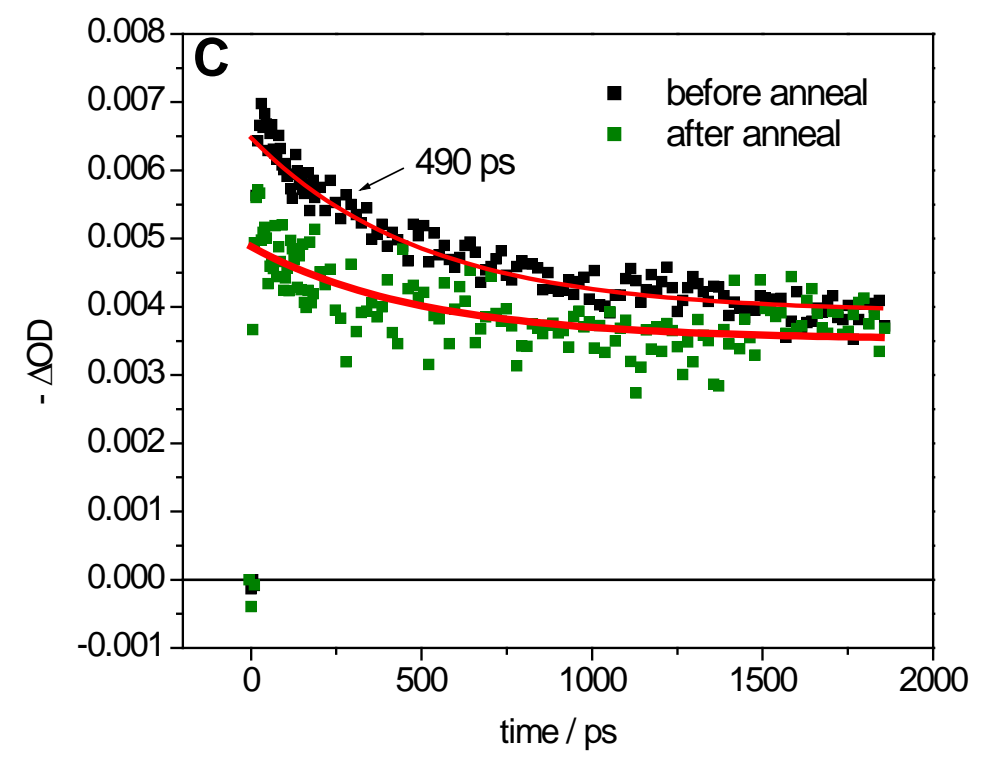

Figure 1. (A) Absorption and PL spectra of CdSe core with $3.60 \mathrm{~nm}$ and CdSe/CdS core/shell QD with $1.20 \mathrm{~nm}$ CdS shell, before and after annealing. The PL intensities are normalized such that the maximum intensity corresponds to the static quantum yield.(B) PL decays before and after annealing. Also shown (red curve) is a single exponential decay of 22 ns. (inset: PL decay curves normalized by the initial amplitude) (C) TA bleach recovery kinetics before and after annealing. Also shown are fit curves with 490 ps decay components of different amplitudes.

Much simpler results are obtained for particles having smaller CdSe cores and therefore smaller strain energy densities. Figure 2A shows the absorption and PL spectra of particles with 2.65 diameter CdSe cores and $1.50 \mathrm{~nm}$ thick CdS shells. In this case, the lowest energy exciton shifts from $517 \mathrm{~nm} 585 \mathrm{~nm}$ upon shell deposition. Elastic continuum calculations give a strain energy density of $0.52 \mathrm{eV} / \mathrm{nm}^{2}$, well below the threshold of $0.59 \mathrm{eV} / \mathrm{nm}^{2}$ for formation of an irregular shell. These particles are in the stable regime of strain energy densities. Figure 2 shows that annealing has essentially no effect on either the static or time resolved spectroscopy. The PL maximum (figure 2A) is unchanged and the PL decay kinetics (figure 2B) are accurately fit by a single exponential decay with time constants of $24 \mathrm{~ns}$, both before and after annealing. As in figure 1, the long-lived PL decay is assigned to the radiative lifetime. Figure $2 \mathrm{C}$ shows that the lowest exciton bleach recovery kinetics from a low-power TA measurement are essentially identical, having a 230 ps decay component before annealing and after annealing. 

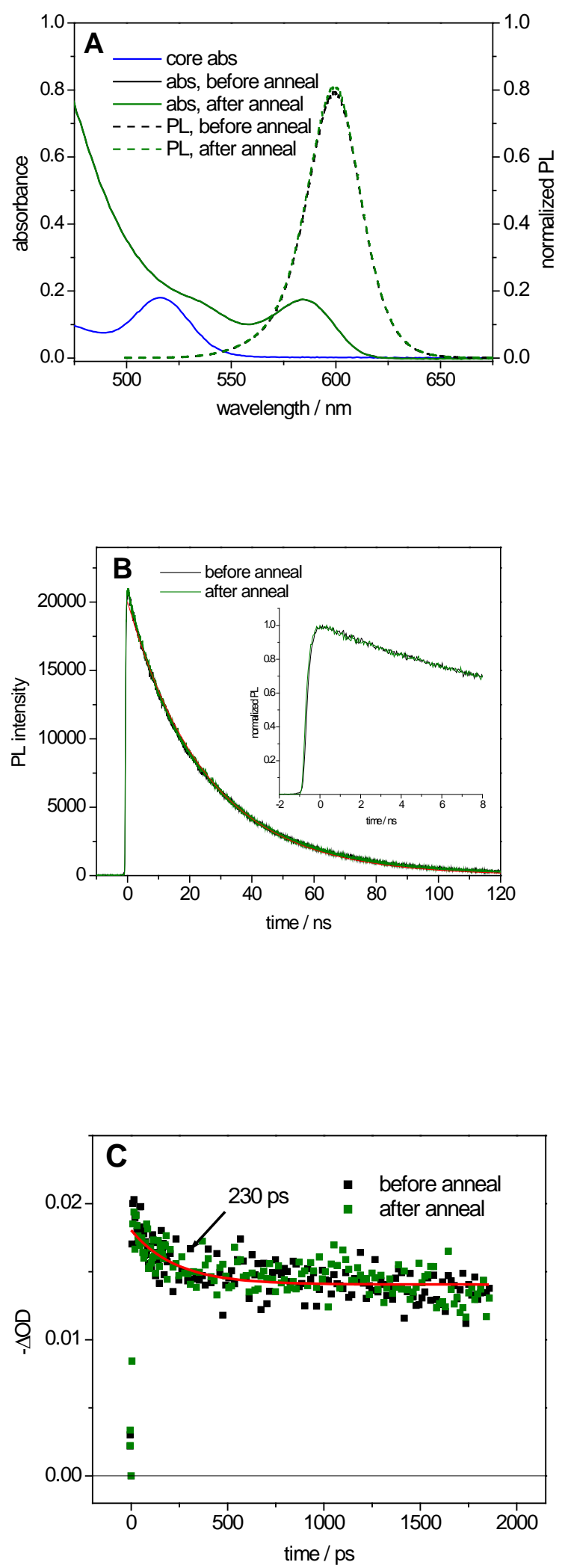
Figure 2. (A) Absorption and PL spectra of $2.65 \mathrm{~nm}$ CdSe core and CdSe/CdS core/shell QDs with $1.50 \mathrm{~nm}$ shell, before and after annealing. The PL intensities are normalized such that the maximum intensity corresponds to the static quantum yield.(B) PL decays before and after annealing. (inset: PL decay curves normalized by the initial amplitude) (C) Bleach recovery of TA kinetics before and after annealing. Also shown are a fit curve with a 230 ps decay component.

The same annealing procedures and subsequent analysis have been applied to several different batches of CdSe/CdS particles having different core sizes and shell thicknesses. In all cases where the strain energy density is in the metastable regime, the data are consistent with those shown in figure 1, see figures $\mathrm{S} 1$ andS2 in the supporting information. In all metastable cases, annealing causes a small red shift of the absorption and PL spectra and an increase in the rates of nonradiative processes, as indicated by a drop in the QY and the presence of short components in the PL decay kinetics. These data are summarized in Table 1, below.

TABLE I. Annealing effect on the spectroscopic properties for metastable and stable particles.

\begin{tabular}{|c|c|c|c|c|c|c|}
\hline $\begin{array}{c}\text { core } \\
\text { diam. } \\
(\mathrm{nm})\end{array}$ & $\begin{array}{c}\text { shell thickness } \\
(\mathrm{nm})\end{array}$ & $\lambda / \mathrm{nm}$ & $\begin{array}{c}\text { energy } \\
\text { density } \\
\left(\mathrm{eV} / \mathrm{nm}^{2}\right)\end{array}$ & $\begin{array}{c}\text { energy } \\
\text { density } \\
\text { regime }\end{array}$ & $\begin{array}{c}\Delta \lambda(\mathrm{nm}) \\
(\text { observed })^{\mathrm{a}}\end{array}$ & $\begin{array}{c}\Delta \lambda(\mathrm{nm}) \\
\left(\text { calculated }^{\mathrm{b}}\right)\end{array}$ \\
\hline 3.40 & 1.40 & 606.0 & 0.66 & metastable & 4.0 & $0.7-7.9$ \\
\hline 3.60 & 1.20 & 604.0 & 0.67 & metastable & 4.0 & $0.7-8.8$ \\
\hline 3.94 & 1.50 & 619.0 & 0.75 & metastable & 3.0 & $1.2-8.7$ \\
\hline 2.65 & 1.50 & 585.0 & 0.52 & stable & 0.0 & $0.0-4.0$ \\
\hline
\end{tabular}

a The energy density regime is defined by comparison of calculated energy density to the threshold of $0.59 \mathrm{eV} / \mathrm{nm}^{2}$. Above/below the threshold indicated as "metastable/stable".

$\mathrm{b}$ The range of values corresponds to release of core pressure to a value corresponding to a strain energy density of $0.59 \mathrm{eV} / \mathrm{nm}^{2}$, and zero core pressure.

The red shift of the absorption and PL spectra can be understood in terms of a partial loss of core compression. The magnitude of the red shift has been calculated in two limiting cases: a loss of core compression corresponding to a reduction of the strain energy density to the threshold values of $0.59 \mathrm{eV} / \mathrm{nm}^{2}$, and a complete loss of core compression. In the former case, this can be a fairly small change in pressure (the strain energy increases quadratically with the pressure) especially if the strain energy density only slightly exceeds the threshold value. If only that amount of strain is relaxed, the spectral shift is relatively small. Table 1 shows that the observed shifts are between these two limiting cases, annealing relieves the strain to below the threshold value, but not to zero. Since the spectral shift varies linearly with the core pressure, 
these spectral shifts indicate that on the order of $1 / 2$ of the core pressure is relieved by annealing. Similar results were reported in an earlier paper.[13]

It is important to note that despite the dramatic increase in the rates of nonradiative processes, the TA results change only slightly: they show that over the first $2.0 \mathrm{~ns}$, the bleach recovery kinetics show a very similar magnitude constant component and a somewhat smaller recovery component after annealing. These observations are crucial to understanding the change in nonradiative decay mechanisms that occur upon annealing and defect formation.

\section{B. Nonradiative processes in particles before and after annealing}

To understand the above results, we must digress into the spectroscopic signatures of the different types of nonradiative decay mechanisms. There are three common nonradiative exciton recombination pathways in CdSe-based core/shell particles, all of which involve bandgap electrons and holes interacting with trap states. These trap states are presumably associated with the surface or other types of localized defects. These mechanisms are electron trapping,[17] hole trapping[18] and surface charging with Auger recombination,[16, 19] as shown in Figure 3.
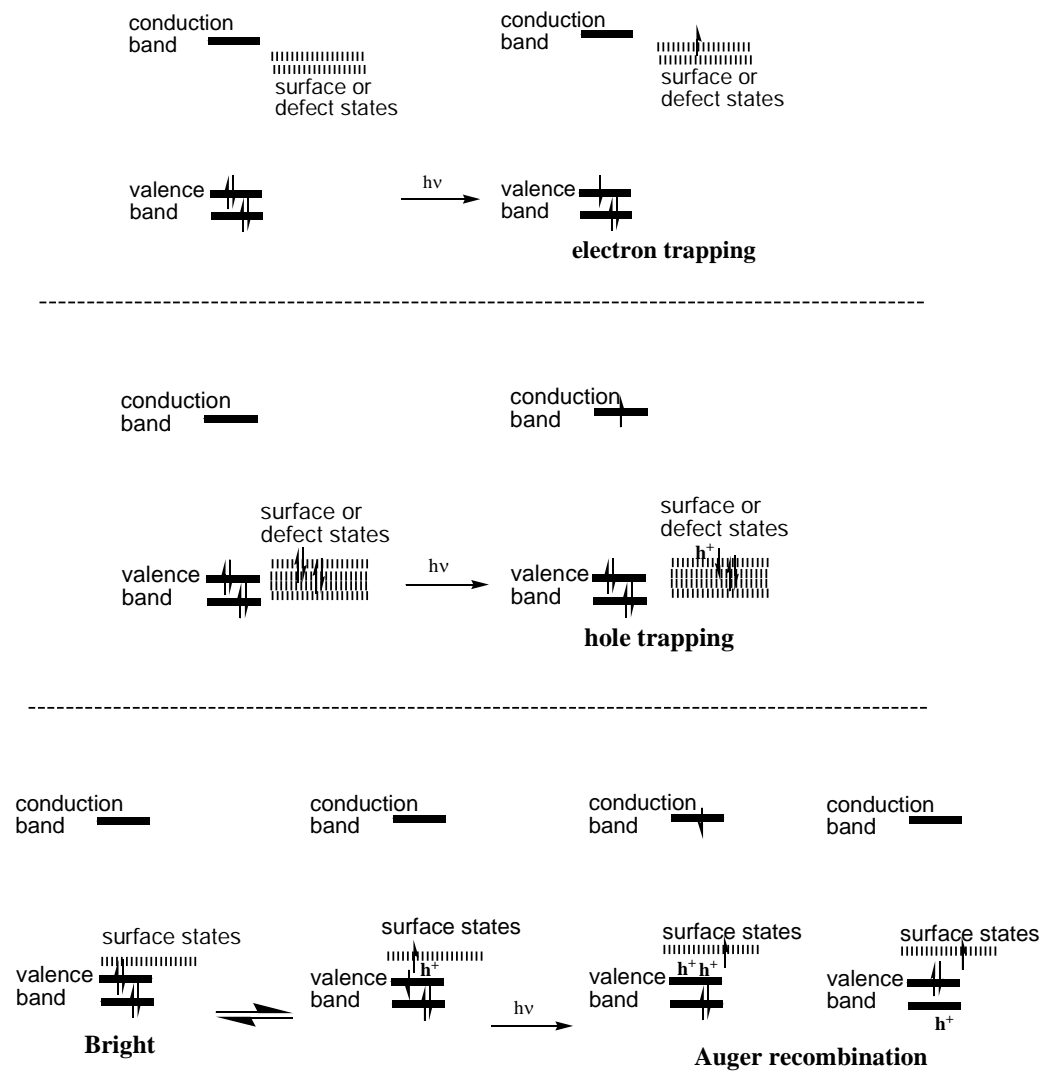
Figure 3. Mechanisms responsible for the radiationless decay of excitons in II-VI QDs. The energy levels associated with electron trapping (top) hole trapping (middle) and surface charging/Auger recombination (bottom) are indicated.

Electrons can trap in unpassivated empty cadmium $5 S$ orbitals just below the conduction band (figure 3, top panel). Similarly, holes can trap in filled, unpassivated chalcogenide 3P (sulfur) or 4P (selenium) orbitals just above the valence band (figure 3, middle panel). Both electron and hole trapping replace one of the delocalized exciton carriers with a trapped carrier that eventually undergoes nonradiative recombination with the remaining carrier. The result is that both electron and hole trapping quench the bandedge luminescence, giving a reduced QY and a PL decay rate that is greater than the radiative rate.

There is another nonradiative process that is particularly important in relatively highQY particles. If unpassivated surface selenium or sulfur orbitals are partially or completely empty and they can be thermally populated by delocalized valence band electrons, see figure 3, bottom panel. When this occurs, the core of the particle is left positively charged - there is a valence band hole. The extent to which this thermal equilibrium produces valence band holes is determined by the energy and density of empty surface chalcogenide orbitals. Photoexcitation of a surface charged particle creates a particle having a conduction band electron and two valence band holes - it is essentially a positive trion. The trions can undergo a fast Auger decay processes, quenching the exciton and creating a hot hole which subsequently relaxes.[20]

It is important to consider which of these nonradiative processes can be detected with time-resolved PL and TA measurements. Time-resolved PL measurements measure the time dependent exciton population - any nonradiative process that depopulates either conduction band electrons or valence band holes will be seen in a time-resolved PL experiment having sufficient time resolution. We have recently shown[16] that consistent with literature reports,[20,21] trion Auger recombination takes place on the tens to a few hundred picoseconds timescale, which is not quite resolvable with our time-correlated photon-counting apparatus. TA (bleach recovery) measurements are sensitive only to the population of electrons in the conduction band[22, 23] and with sub-picosecond time resolution, can therefore detect all but the fastest conduction band dynamics. The conclusions in terms of the relaxation mechanisms in figure 3 are that time-resolved PL measures all three processes, but only on a timescale longer than the Auger processes and that only hole trapping results in no transient absorption signal. Thus, the Auger recombination rate in surface charged particles can be measured in the TA experiment and can be distinguished from hole trapping only by the characteristic timescale of the Auger processes.

Figure 1B shows that the particles before annealing have a QY of $78 \%$ and almost single exponential PL decay kinetics. With the assignment that the $22 \mathrm{~ns}$ decay corresponds to the 
radiative lifetime, these results indicate that the bright particles have a QY that is close to unity and that about $22 \%$ of the particles are dark, having a lifetime of less than a few hundred picoseconds. The process that makes this fraction of the particles dark is elucidated by the transient absorption results in figure 1C. It shows that about $25 \%$ of the conduction band electrons are depleted with a time constant of $\sim 490$ ps. This lifetime is in good agreement with that expected for an Auger process. In previous work[16] we showed that trion Auger times in core/shell particles scale almost linearly with particle "effective volume", defined as the volume of a CdSe particle having the same exciton energy as the core/shell particle.[24] Figure 4 shows that the present results follow that same trend. We conclude that prior to annealing about $22 \%$ of the particles are dark because of Auger recombination following thermal surface charging.

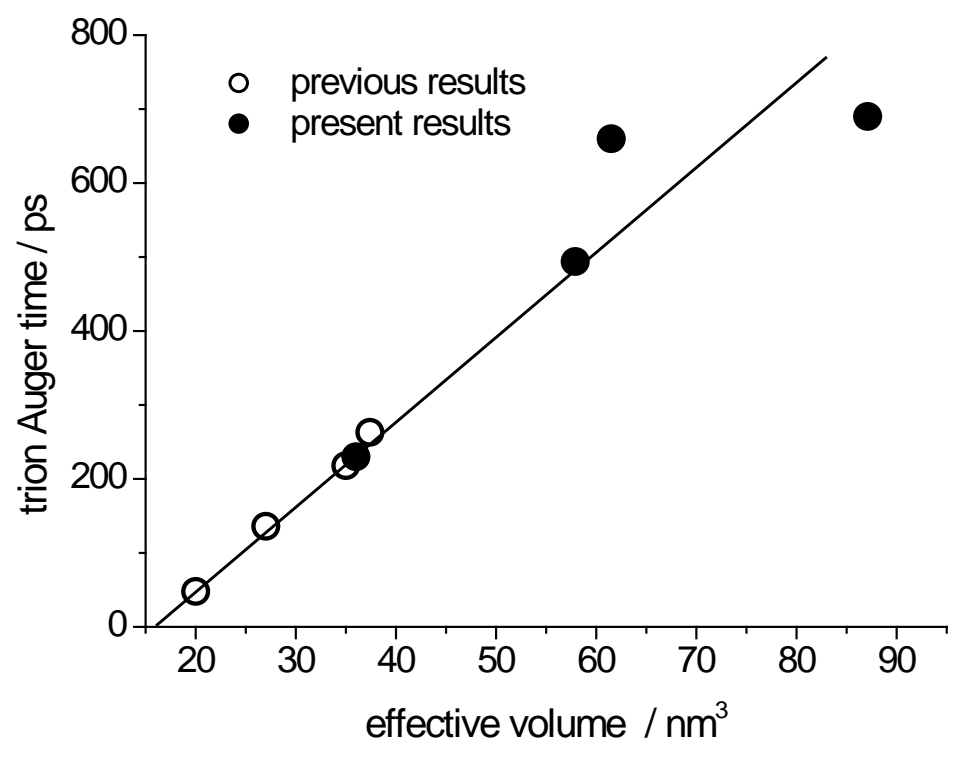

Figure 4. Plots of positive trion Auger times (open circles) from a previous report[16] and the present study (solid circles). The trion time increases approximately linearly with the effective volume (straight line).

Figure 1A shows that after annealing, the QY declines by a factor of about 5. The PL kinetics in figure $1 \mathrm{~B}$ show that the decay becomes somewhat faster, especially in the first $10 \mathrm{~ns}$, due to the formation of electron and/or hole traps. However, the largest effect on the PL decay is the dramatic decrease in intensity. This decrease is seen in both the intensity of the PL decay curves and the static PL spectra, and indicates that annealing causes a large increase in the fraction of particles that are dark. The comparison of the low-power TA bleach kinetics allows determination of the additional nonradiative mechanisms brought about by annealing. Figure 
1C shows that the initial magnitude of the bleach is only slightly smaller than that observed before annealing and that the bleach recovery kinetics show the same 490 ps decay component, having a $30 \%$ smaller amplitude. Because the TA signal measures only the conduction band population, this indicates that despite the factor of 5 reduction in exciton population (as indicated by the PL results), the conduction band population is reduced only slightly, compared to the before annealing case. The only possibility is that the exciton population is reduced by loss of the hole population, i.e., hole trapping. The hole trapping timescale is not addressed by the TA experiment and can only be determined to take place on a timescale that is not resolved in the PL decay kinetics, less than a few hundred picoseconds. Similar results have been obtained for other core/shell particles in the metastable regime (Table I and Figure 4) and are given in the supporting information. In the case of the particles having the largest effective volumes, the Auger recombination prior to annealing is marginally resolvable in the PL decays, see figure S1 in the supporting information.

\section{Comparison from Transmission Electron Microscopy.}

Previous work has demonstrated that annealing of metastable core/shell particles will roughen the surfaces, which creates shell thickness inhomogeneities, and increases the total surface area of the particles. Figure 5 examines whether these changes can be observed in highresolution TEM. TEM images of the particles corresponding to figure 1 show that some particle aggregation of the particles occurs on the TEM grid, primarily along the tetrahedrally oriented (111) facets, see Figure 5 and Figure S4 in the supporting information.
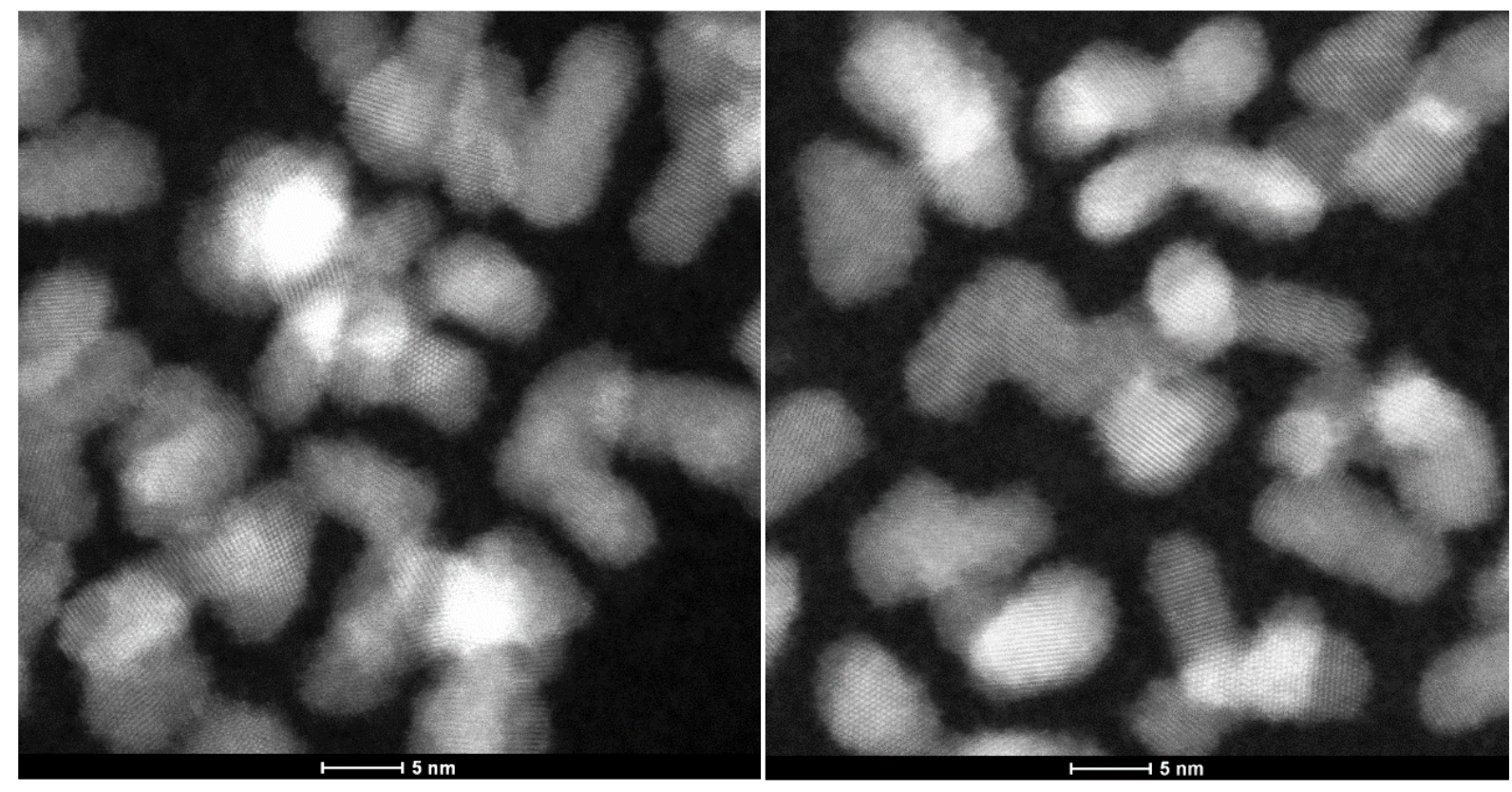


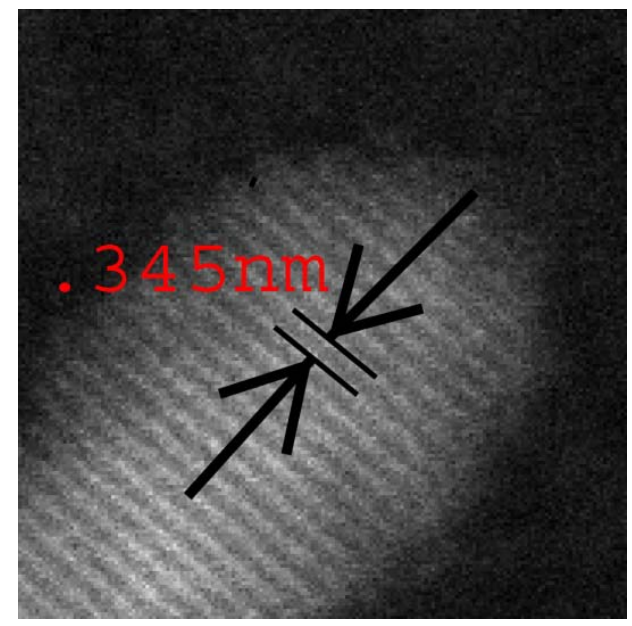

Figure 5. High resolution TEM images of CdSe/CdS core/shell QDs with $3.60 \mathrm{~nm}$ CdSe core and $1.20 \mathrm{~nm}$ CdS shell. (left: before annealing right: after annealing) Also shown is the (111) lattice fringe spacing taken from a particle before annealing (lower).

The lattice (111) lattice fringe spacings for particles prior to annealing have been measured and give a spacing of about $0.345 \mathrm{~nm}$, which is about $1.7 \%$ below the literature value of $0.351 \mathrm{~nm}$.[25] We find that this varies slightly ( $\pm 1 \%$ ) from particle to particle. The difference between core/shell nanocrystal and bulk values is a small, but significant and can be understood in terms of compression of the CdSe core. For these core/shell particles the CdSe core is calculated to have an isotropic pressure of $1.87 \mathrm{GPa}$. CdSe has a bulk modulus of $53.3 \mathrm{GPa}$,[25] and this pressure corresponds to a calculated volume compression of $3.5 \%$, or about $1.1 \%$ linear compression. This is in agreement with the measured lattice fringe spacing, within the accuracy of these measurements. A similar correspondence has been observed in CdTe/CdSe core/shell particles.[26] Strain release upon annealing should reduce the extent of compression. The spectroscopic results in Table 1 indicate that only partial (about half) strain release occurs upon annealing. In principle, it should be possible to determine the extent of strain release from the lattice fringe spacings. However, there is enough uncertainty in the lattice fringe spacings that this difference could not be reliably measured.

Despite aggregation on the TEM grid, the images in figure 5 show similar size and morphology for the samples before and after annealing. Figure 5 also shows that no change in the zincblende crystal structure is observed upon annealing. This excludes the possibility that the QY drop after annealing is due to a transformation from zincblende to wurtzite in both core and shell, in which core/shell particles with wurtzite crystal structure usually have lower QY than the zincblende counterpart.[14] This is consistent with literature reports showing that ligand-surface interactions and the ligand environment play dominant roles in determining the 
crystal structure.[27] Furthermore, it shows that annealing results in no obvious stacking faults due to the accumulation of dislocations or point defects.

\section{CONCLUSIONS}

The results presented here demonstrate that annealing at $210-220{ }^{\circ} \mathrm{C}$ results in dramatic changes in the nonradiative dynamics of CdSe/CdS core/shell particles having strain energy densities in the metastable regime $\left(0.59-0.85 \mathrm{eV} / \mathrm{nm}^{2}\right)$, and that these dynamics play a dominant role in the one-photon photophysics. Annealing creates defects due to the release of strain at the core/shell interface and thus decreases the QY. Based on PL decay and transient absorption bleach recovery kinetics, we conclude that prior to annealing, the small amount of nonradiative decay is almost entirely due to surface charging and Auger recombination. Subsequent annealing results in the formation of hole trapping defects, which is then the dominant nonradiative decay process. Even though annealing results in the formation of defects that dramatic change the optical properties, electron microscopy does not show any fundamental changes in particle morphology, crystal structure or the presence of stacking faults.

\section{ACKNOWLEDGEMENTS.}

This material is based upon work supported by the U.S. Department of Energy, Office of Science, Office of Basic Energy Sciences, under award number FG02-13ER16371. The authors would also like to thank Dr. James E. Martin, Dr. Lauren E. Shea-Rohwer, and Dr. Ping Lu of Sandia National Labs for their collaboration with the TEM imaging.

Supporting Information Available. Synthetic methods, optical measurement details and absorption and photoluminescence spectra are given in the Supporting Information.

\section{REFERENCES.}

[1] D.V. Talapin, I. Mekis, S. Gotzinger, A. Kornowski, O. Benson, H. Weller, J. Phys. Chem. B, 108 (2004) 18826-18831.

[2] P. Reiss, J. Bleuse, A. Pron, Nano Lett., 2 (2002) 781-784.

[3] D.V. Talapin, A.L. Rogach, A. Kornowski, M. Haase, H. Weller, Nano Lett., 1 (2001) 207.

[4] X. Peng, M.C. Schlamp, A.V. Kadavanich, A.P. Alivisatos, J. Am. Chem. Soc., 119 (1997) 7019 $-7029$.

[5] K. Gong, Y. Zeng, D.F. Kelley, J. Phys. Chem. C, 117 (2013) 20268-20279.

[6] K. Gong, D.F. Kelley, J. Chem. Phys., 141 (2014) 194704 - 194712.

[7] Z.-J. Jiang, D.F. Kelley, J. Phys. Chem. C, 116 (2012) 12958 -12968.

[8] Z.-J. Jiang, D.F. Kelley, J. Phys. Chem. C, 117 (2013) 6826 - 6834. 
[9] A.S. Saada, Elasticity Theory and Applications, Permagon Press, New York, 1974.

[10] Y. Guo, K. Marchuk, S. Sampat, R. Abraham, N. Fang, A.V. Malko, J. Vela, J. Phys. Chem. C 116 (2012) 2791-2800.

[11] Y. Chen, J. Vela, H. Htoon, J.L. Casson, D.J. Werder, D.A. Bussian, V.I. Klimov, J.A. Hollingsworth, J. Am. Chem. Soc., 130 (2008) 5026-5027.

[12] A. Cretí, M. Zavelani-Rossi, G. Lanzani, M. Anni, L. Manna, M. Lomascolo, Phys. Rev. B, 43 (2006) 165410.

[13] K. Gong, D.F. Kelley, J. Phys. Chem. Lett., 6 (2015) 1559-1562.

[14] W. Nan, Y. Niu, H. Qin, F. Cui, Y. Yang, R. Lai, W. Lin, X. Peng, J. Am. Chem. Soc. , 134 (2012) 19685-19693.

[15] K. Gong, J.E. Martin, L.E. Shea-Rohwer, P. Lu, D.F. Kelley, J. Phys. Chem. C, 119 (2015) 2231-2238.

[16] K. Gong, D.F. Kelley, J. Phys. Chem. C, 119 (2015) 9637-9645.

[17] S.J. Kern, K. Sahu, M.A. Berg, Nano Lett., 11 (2011) 3493-3498.

[18] F.M. Gómez-Campos, M. Califano, Nano Lett., 12 (2012) 4508-4517.

[19] X. Cai, J.E. Martin, L.E. Shea-Rohwer, K. Gong, D.F. Kelley, J. Phys. Chem. C, 117 (2013) $7902-7913$.

[20] Y.-S. Park, W.K. Bae, J.M. Pietryga, V.I. Klimov, ACS Nano, 8 (2014) 7288-7296.

[21] D.E. Gómez, J.v. Embden, P. Mulvaney, M.J. Fernée, H. Rubinsztein-Dunlop, ACS Nano, 3 (2009) 2281-2287.

[22] V.I. Klimov, Annu. Rev. Phys. Chem. , 58 (2007) 635-673.

[23] V.I. Klimov, D.W. McBranch, Phys. Rev. Lett., 80 (1998) 4028.

[24] F. García-Santamaría, S. Brovelli, R. Viswanatha, J.A. Hollingsworth, H. Htoon, S.A. Crooker, V.I. Klimov, Nano Lett., 11 (2011) 687-693.

[25] A.R. West, Basic Solid State Chemistry, Wiley Chichester, 1988.

[26] X. Cai, H. Mirafzal, K. Nguyen, V. Leppert, D.F. Kelley, J. Phys. Chem. C, 116 (2012) 8118 8127.

[27] Y. Gao, X. Peng, J. Am. Chem. Soc., 136 (2014) 6724-6732. 

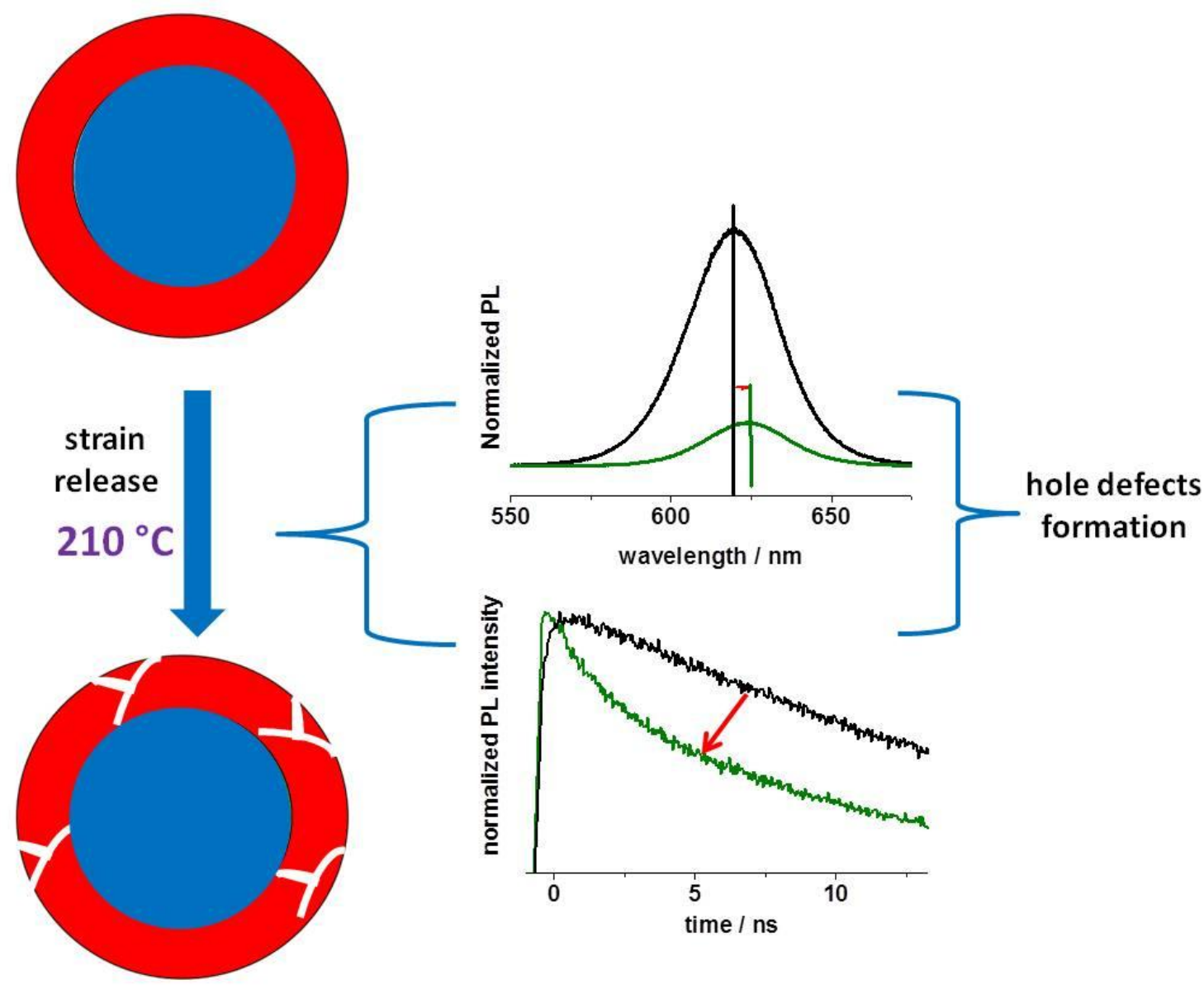